\title{
Signaling Networks Controlling HCC Onset and Progression: Influence of Microenvironment and Implications for Cancer Gene Therapy
}

\author{
Alessandra Marchetti, Carla Cicchini, Laura Santangelo, Angela Maria Cozzolino, Viviana Costa, \\ Marco Tripodi*, Laura Amicone \\ Pasteur Institute-Cenci Bolognetti Foundation, Department of Cellular Biotechnologies and Hematology, Sapienza University, \\ Rome, Italy. \\ Email: "tripodi@bce.uniroma1.it
}

Received December $19^{\text {th }}, 2012$; revised January $22^{\text {nd }}, 2013$; accepted January $31^{\text {st }}, 2013$

\begin{abstract}
Hepatocarcinogenesis, as other epithelial malignancies, has been proved to be a multistep process that, starting from mutagenic events, allows the transformed liver cell to evolve towards a more aggressive phenotype, characterized by the acquisition of migratory/invasive and stem-cell-like properties. Hepatocellular carcinoma (HCC) can originate from both mature hepatocytes and liver precursor/stem cells. Whatever its origin, a common feature of advanced-stage HCC is the reduction or lack of expression of master genes of epithelial/hepatocyte differentiation, i.e. members of the liver enriched transcription factors (LEFTs) family like HNF4 $\alpha$, and conversely an increased expression of epithelialto-mesenchymal transition (EMT) master genes, i.e. the transcriptional repressors belonging to the Snail family. Recently, it has emerged as members of these families are capable to directly repress each other and to regulate in opposite manner target genes involved in stemness and in hepatocyte differentiation, thus influencing cell outcome between epithelial/differentiated/poor aggressive and mesenchymal/undifferentiated/aggressive phenotype. Consequently, the restoration of LEFT functions in invasive HCC could represent an important goal for anti-cancer therapies. However, any strategy based on gene transfer needs to take in account the influence of micro-environmental factors in HCC tumor niche, like TGF $\beta$, responsible for shifting the described balance in tumor cell towards the acquisition of stem-cell like properties and invasiveness, through Snail/EMT induction and LEFTs downregulation. The presence of this cytokine, indeed, was shown to override both anti-EMT and tumor suppressor activity of the ectopically expressed HNF4 $\alpha$ protein. In this review, the rationale to propose implementation of HCC gene therapy will be discussed.
\end{abstract}

Keywords: HNF4; Snail; TGF $\beta$; microRNA; EMT; Cancer Stem Cell

\section{Introduction}

Hepatocellular carcinoma (HCC) is the prevalent worldwide cancer and the most common type of liver cancer originating from hepatocytes, showing a high mortality for ineffective current therapies. Progression of the malignant phenotype in HCC is associated with increased proliferation, epithelial/hepatic de-differentiation, acquisition of invasive and metastatic properties [1].

Identification of molecular pathways that drive HCC development is an important challenge for the design of successful therapies based on the restoration of multilevel controls of proliferation/differentiation, lost during neoplastic transformation. The knowledge of molecular signature in HCC cells could provide, therefore, new targets and/or new tools for therapeutic approaches in vivo.

*Corresponding author.
A variety of genomic and molecular alterations have been detected in fully developed HCC [1,2]. In particular, while well-differentiated HCC tumors show E-cadherin expression, its plasmamembrane localization and expression of liver enriched transcription factors (LEFTs), like HNF4 $\alpha$, poorly differentiated HCCs present disruption of cell-to-cell junctions, E-cadherin delocalization and fail to express $\mathrm{HNF} 4 \alpha$. Importantly, the differentiation grade of HCC is inversely correlated to a poor prognosis [3]. These observations correlate HCC progression with the activation of an epithelial-to-mesenchymal transition (EMT) [4], the transdifferentiation process that results in the loss of epithelial polarity and identity and in the acquisition of a mesenchymal phenotype and motility.

EMT can play an important role in determining the dissemination of tumors [5], while the reverse process mesenchymal-to-epithelial transition (MET) seems to oc- 
cur when invasive cancer cells, in secondary sites within distant metastasis, re-express some epithelial markers. In addition, EMT might provide cells with stem cell properties [6]. Acquisition of stemness traits during EMT, in particular, can contribute to the described intra-tumoral heterogeneity resulting in cell subpopulations, characterized by different stages of epithelial/mesenchymal differentiation [7].

A number of genes have been identified to play a causal role in EMT. Among these genes, the role of the transcription factors belonging to Snail family in cancer-associated EMT has been highlighted in a variety of histotypes [8], including hepatocytes [9]. Responding to environmental cues, Snail family members (e.g. Snail and Slug) act as master regulators, altering the expression of a broad number of genes, including E-cadherin [8,10].

In addition, it has been highlighted that these repressors cause the down-regulation of a number of micro RNAs, an emerging pivotal category of molecules capable to negatively and specifically regulate gene expression. The liver-specific microRNA miR-122, already characterized as a potent tumor suppressor, has been found downregulated in metastatic HCC [11]. Similarly, miR200 and miR-34 family members, targeting Zeb1/2 and Snail, are described as critical negative regulators of EMT in tumor progression [12,13]. Interestingly, it has been recently described their positive regulation by p53 in inducing tumor suppression of several neoplasia [1315], including HCC [16], thus suggesting the activation of p53-miRNAs pathway as potential therapeutic strategy.

\section{The Balance of EMT/MET Players: The Route for the Invasiveness or Tumor Suppression}

EMT is a phenomenon shared by a variety of epithelial cells. Several signal transduction pathways triggering EMT converge on the transcriptional inhibitor Snail, inducing its expression or stabilizing the protein by interference with its ubiquitination/degradation [17].

Snail is a well-known transcriptional repressor of Ecadherin gene [18]. In hepatocytes, the action of Snail is conveyed also to tissue-specific functions since it is able to directly repress the expression of $\mathrm{HNF} 4 \alpha$ through the binding to its promoter [9]. In the liver, therefore, the Snail-mediated EMT is accomplished not only by inhibition of the epithelial program but, more specifically, by direct interference with the program of hepatocyte differentiation. Notably, a regulatory mini-circuitry of reciprocal repression has been recently unveiled: it has been demonstrated that HNF4 $\alpha$ acts as a direct repressor of Snail and other master regulators of the EMT program, such as Slug and HMGA2. Moreover, a stable inhibitory binding of HNF4 $\alpha$ on regulatory sequences of mesenchymal genes was found in differentiated hepatocytes. Simi- lar function has been attributed to HNF1 $\alpha$, other member of LEFT family $[19,20]$.

The silencing of HNF4 $\alpha$ in cultured hepatocytes as well as in KO murine models causes the loss of cell polarity together with the switch on of a mesenchymal program [19]. These observations have allowed to attribute to HNF4 $\alpha$ the important new role of negative regulator of the mesenchymal program in hepatocytes, essential for a full execution and for the stable maintenance of the epithelial program.

Thus, the simple cross-regulatory circuit between Snail and HNF4 $\alpha$, causing a mutually exclusive expression due to the reciprocal direct repression, is responsible for the dynamic balance of EMT/MET.

In Garibaldi et al. [21], it is unveiled a further role for this molecular circuitry in the control of liver stem cell plasticity. Starting from the finding that Snail is expressed in resident liver stem cell lines [22], it has been demonstrated a positive role of this transcriptional factor in the expression of stemness markers. This observation was unexpected considering that the transcriptional repression is the only function so far attributed to Snail. Investigating on other players integrating/mediating Snail activity, Snail was shown to inhibit the hepatospecific program through direct repression of the epithelial miR200c and 34a genes, microRNAs that in turn target mRNAs of several stemness genes. In differentiated hepatocytes, on the other hand, HNF4 $\alpha$, together with a direct repression of Snail gene, directly upregulates miR-200 family members (200 a, b, c) and miR-34a, thus further stabilizing the differentiated phenotype. Altogether, these data unveiled Snail, HNF4 $\alpha$ and miRNAs (miR-200 family and miR-34a) as epistatic elements controlling hepatic stem cell maintenance/differentiation [21].

\section{Microenvironmental Cues in HCC: The Role of TGF $\boldsymbol{\beta}$}

Tumor onset and progression depend not only on the acquisition of genetic and/or epigenetic mutations by hepatocytes or hepatic stem/precursor cells but also on microenvironmental cues influencing tumor niche.

The pleiotropic transforming growth factor $\beta$ (TGF $\beta$ ) has emerged as a major microenvironmental factor playing a role in carcinoma progression. In fact, in spite of its tumor suppressor activity in the early-phase of tumorigenesis, where it induces growth arrest and apoptosis, TGF $\beta$ appears to take on the opposite role in end-stage tumors, where it promotes metastasis through different mechanisms [23]. In HCC, the TGF $\beta$ signaling is often found constitutively activated [24]. HCC patients showed a high plasma level of TGF $\beta$ [25] that has been correlated to an increased tumor vascularization [26]. Moreover, a significant intracellular expression of TGF $\beta$, sustaining an autocrine loop, has been described $[27,28]$. 
Thus, both intrinsic and extrinsic TGF $\beta$ signaling activation contributes to in vivo HCC progression and is associated to a poor prognosis [24]. Understanding the molecular mechanisms through which tumor cells respond to TGF $\beta$ and switch their response to this cytokine from tumor arrest to tumor progression is pivotal for the development of TGF $\beta$ targeted therapeutics.

Dissection of TGF $\beta$ signaling in HCC cell lines showed that it promotes tumor progression mainly by 1 ) inducing EMT through the up-regulation of EMT master genes (i.e. Snail and Slug); 2) inhibiting cell differentiation and 3) causing the acquisition of stem-cell like phenotype [2931]. Furthermore, autocrine TGF $\beta$ production enhances angiogenesis in close proximity to the tumor, supplying a route for tumor cell migration [23,32], and confers resistance to anoikis allowing the survival of tumor cells in the bloodstream [33].

The TGF $\beta$-induced EMT has been largely described. The cytokine is one of the major inducer of EMT during development and in tumors, through the activation of Smad-dependent and independent signaling pathways [29, 34]. EMT can be initiated by TGF $\beta$ through autocrine or paracrine activation of intracellular signaling that triggers reprogramming of the epithelial cell both at transcriptional [35,36] and post-translational level [37]. A common element in the induction of EMT by TGF $\beta$ is the Snail gene upregulation [38] and Snail protein stabilization by specific post-translational modifications $[39,40]$.

In hepatocytes and HCC cell lines, the TGF $\beta$ treatment was found to induce a switch in the above described regulatory circuitry Snail/Hnf $4 \alpha /$ miRNAs through 1) Snail gene upregulation and consequent direct and miRNAmediated induction of EMT genes [19,21] and repression of HNF4 $\alpha$ [9] and of epithelial molecules (i.e. E cadherin, occludin, claudin) $[9,18]$; 2) transcriptional down-regulation of HNF $4 \alpha$ preceded by the loss of its inhibitory binding to Snail promoter and its activating binding on promoters of genes controlling liver functions $[9,41]$.

Thus, an unbalanced level of TGF $\beta$ in the stem-cell niche can result in sustained self-renewal of liver stem cells, in an impairment of their hepatocytic differentiation program and, ultimately, in a high risk of accumulating mutations; in the tumor environment, moreover, the cytokine can drive transformed hepatocytes towards an epithelial-to-mesenchymal transition and, consequently, the acquisition of migration and invasive properties.

\section{HNF4 $\alpha$ as Gene Therapy Tool for HCC}

As discussed above, an inverse regulation between Snail and HNF4 $\alpha$ is observed during HCC progression. Snail expression significantly increases along with hepatocellular carcinoma dedifferentiation, accelerating cancer invasion and resulting in poor prognosis [42]. On the other hand, the loss of HNF4 expression is an important determinant of HCC onset and progression [43] and its re-expression has been showed to promote reversion towards a highly differentiated and less invasive phenotype. HNF4 $\alpha$, in fact, is able to repress EMT program [19] and to promote hepatocyte differentiation [44]; moreover, HNF4 $\alpha$ overexpression in hepatocytes of fibrotic livers counteracts the production of fibrogenic cells, ameliorating the fibrosis and thus preventing its most feared complication, the HCC $[45,46]$. Therefore, restoration of HNF4 $\alpha$ functions in invasive HCC represents an important goal for anti-cancer therapies.

However, recent data showed that in a TGF $\beta$-containing environment, the restoration of HNF4 $\alpha$ function does not suppress TGF $\beta$-induced tumor promoting effects; the cytokine appears, indeed, dominant on HNF4 $\alpha$ activity, thus indicating that the therapeutic use of HNF $4 \alpha$ gene delivery is limited by the presence of TGF $\beta$ in the tumor environment. In particular, TGF $\beta$ signaling was found to induce several post-translational modifications that in turn determine its displacement from target gene promoters, including Snail [41].

\section{Conclusions and Perspective}

The balance between Snail (EMT master gene) and HNF $4 \alpha$ (MET master gene), in liver stem cells and in liver cancer cells (cancer stem cells or transformed hepatocytes) ultimately influences the outcome of the transition between the mesenchymal/undifferentiated and the epithelial/differentiated phenotype. The epistatic relation among these transcriptional factors, a number of miRNAs, and their target genes, is further influenced by environmental cues such as TGF $\beta$ (Figure 1).

The hierarchical relevance of these master regulatory molecules, controlling a broad range of cellular functions, allows to design simple molecular therapies based on a gene transfer approach.

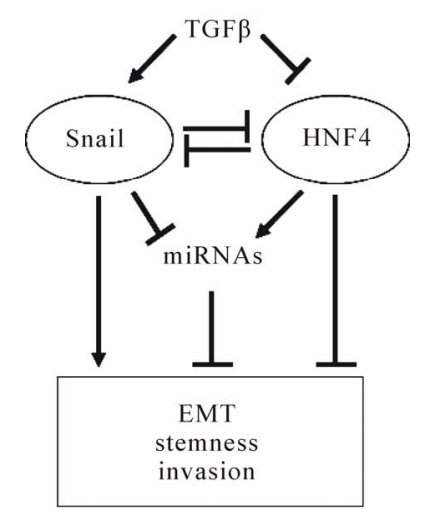

Figure 1. Epistatic circuitry controlling tumor cell outcome. Microenvironmental factors, like TGF $\beta$, influence balance between snail (EMT master gene) and HNF4 $\alpha$ (MET master gene) inducing tumor progression. 
This strategy, even though promising in in vitro and in preclinical studies, should take in account for in clinical applications the tumor niche influence, capable to exert direct functional impairment of therapeutic molecules.

In this context, the use of engineered molecular tools, insensitive to negative regulation by the microenvironment, will represent the successful approach to improve gene therapy strategies.

\section{REFERENCES}

[1] S. S. Thorgeirsson and J. W. Grisham, "Molecular Pathogenesis of Human Hepatocellular Carcinoma,” Nature Genetics, Vol. 31, No. 4, 2002, pp. 339-346. doi:10.1038/ng0802-339

[2] S. Imbeaud, Y. Ladeiro and J. Zucman-Rossi, "Identification of Novel Oncogenes and Tumor Suppressors in Hepatocellular Carcinoma," Seminars in Liver Disease, Vol. 30, No. 1, 2010, pp. 75-86. doi:10.1055/s-0030-1247134

[3] K. Endo, T. Ueda, J. Ueyama, T. Ohta and T. Terada, "Immunoreactive E-Cadherin, Alpha-Catenin, Beta-Catenin, and Gamma-Catenin Proteins in Hepatocellular Carcinoma: Relationships with Tumor Grade, Clinicopathologic Parameters, and Patients' Survival," Human Pathology, Vol. 31, No. 5, 2000, pp. 558-565. doi:10.1053/hp.2000.6683

[4] F. van Zijl, G. Zulehner, M. Petz, D. Schneller, C. Kornauth, M. Hau, et al., "Epithelial-Mesenchymal Transition in Hepatocellular Carcinoma," Future Oncology, Vol. 5, No. 8, 2009, pp. 1169-1179. doi:10.2217/fon.09.91

[5] J. P. Thiery, "Epithelial-Mesenchymal Transitions in Tumour Progression,” Nature Reviews Cancer, Vol. 2, No. 6, 2002, pp. 442-454. doi:10.1038/nrc822

[6] T. Brabletz, F. Hlubek, S. Spaderna, O. Schmalhofer, E. Hiendlmeyer, A. Jung, et al., "Invasion and Metastasis in Colorectal Cancer: Epithelial-Mesenchymal Transition, Mesenchymal-Epithelial Transition, Stem Cells and Beta-Catenin,” Cells, Tissues, Organs, Vol. 179, No. 1-2, 2005, pp. 56-65. doi:10.1159/000084509

[7] K. Polyak and R. A. Weinberg, "Transitions between Epithelial and Mesenchymal States: Acquisition of Malignant and Stem Cell Traits," Nature Reviews Cancer, Vol. 9, No. 4, 2009, pp. 265-273. doi:10.1038/nrc2620

[8] M. A. Nieto, “The Snail Superfamily of Zinc-Finger Transcription Factors,” Nature Reviews Molecular Cell Biology, Vol. 3, No. 3, 2002, pp. 155-166. doi:10.1038/nrm757

[9] C. Cicchini, D. Filippini, S. Coen, A. Marchetti, C. Cavallari, I. Laudadio, et al., "Snail Controls Differentiation of Hepatocytes by Repressing HNF4alpha Expression,” Journal of Cellular Physiology, Vol. 209, No. 1, 2006, pp. 230-238. doi:10.1002/jcp.20730

[10] A. Cano, M. A. Perez-Moreno, I. Rodrigo, A. Locascio, M. J. Blanco, M. G. del Barrio, et al., "The Transcription Factor Snail Controls Epithelial-Mesenchymal Transitions by Repressing E-Cadherin Expression," Nature Cell Boilogy, Vol. 2, No. 2, 2000, pp. 76-83.

doi:10.1038/35000025
[11] W. C. Tsai, P. W. Hsu, T. C. Lai, G. Y. Chau, C. W. Lin, C. M. Chen, et al., "MicroRNA-122, a Tumor Suppressor microRNA That Regulates Intrahepatic Metastasis of Hepatocellular Carcinoma,” Hepatology, Vol. 49, No. 5, 2009, pp. 1571-1582. doi:10.1002/hep.22806

[12] S. M. Park, A. B. Gaur, E. Lengyel and M. E. Peter, "The miR-200 Family Determines the Epithelial Phenotype of Cancer Cells by Targeting the E-Cadherin Repressors ZEB1 and ZEB2," Genes \& Development, Vol. 23, No. 11, 2008, pp. 894-907. doi:10.1101/gad.1640608

[13] H. Siemens, R. Jackstadt, S. Hunten, M. Kaller, A. Menssen, U. Gotz, et al., "miR-34 and SNAIL form a DoubleNegative Feedback Loop to Regulate Epithelial-Mesenchymal Transitions," Cell Cycle, Vol. 10, No. 24, 2011, pp. 4256-4271. doi:10.4161/cc.10.24.18552

[14] C. J. Chang, C. H. Chao, W. Xia, J. Y. Yang, Y. Xiong, C. W. Li, et al., "p53 Regulates Epithelial-Mesenchymal Transition and Stem Cell Properties through Modulating miRNAs,” Nature Cell Biology, Vol. 13, No. 3, 2011, pp. 317-323. doi:10.1038/ncb2173

[15] N. H. Kim, H. S. Kim, X. Y. Li, I. Lee, H. S. Choi, S. E. Kang, et al., "A p53/miRNA-34 Axis Regulates Snail1Dependent Cancer Cell Epithelial-Mesenchymal Transition,” The Journal of Cell Biology, Vol. 195, No. 3, 2011, pp. 417-433. doi:10.1083/jcb.201103097

[16] T. Kim, A. Veronese, F. Pichiorri, T. J. Lee, Y. J. Jeon, S. Volinia, et al., "p53 Regulates Epithelial-Mesenchymal Transition through MicroRNAs Targeting ZEB1 and ZEB2,” The Journal of Experimental Medicine, Vol. 208, No. 5, 2011, pp. 875-883. doi:10.1084/jem.20110235

[17] M. A. Huber, N. Kraut and H. Beug, "Molecular Requirements for Epithelial-Mesenchymal Transition during Tumor Progression," Current Opinion in Cell Biology, Vol. 17, No. 5, 2005, pp. 548-558. doi:10.1016/j.ceb.2005.08.001

[18] E. Batlle, E. Sancho, C. Franci, D. Dominguez, M. Monfar, J. Baulida, et al., "The Transcription Factor Snail Is a Repressor of E-Cadherin Gene Expression in Epithelial Tumour Cells,” Nature Cell Biology, Vol. 2, No. 2, 2000, pp. 84-89. doi:10.1038/35000034

[19] L. Santangelo, A. Marchetti, C. Cicchini, A. Conigliaro, B. Conti, C. Mancone, et al., "The Stable Repression of Mesenchymal Program Is Required for Hepatocyte Identity: A Novel Role for Hepatocyte Nuclear Factor 4alpha," Hepatology, Vol. 53, No. 6, 2011, pp. 2063-2074. doi:10.1002/hep. 24280

[20] L. Pelletier, S. Rebouissou, D. Vignjevic, P. Bioulac-Sage and J. Zucman-Rossi, "HNF1alpha Inhibition Triggers Epithelial-Mesenchymal Transition in Human Liver Cancer Cell Lines,” BMC Cancer, Vol. 11, 2011, p. 427. doi:10.1186/1471-2407-11-427

[21] F. Garibaldi, C. Cicchini, A. Conigliaro, L. Santangelo, A. M. Cozzolino, G. Grassi, et al., "An Epistatic Mini-Circuitry between the Transcription Factors Snail and HNF4Alpha Controls Liver Stem Cell and Hepatocyte Features Exhorting Opposite Regulation on Stemness-Inhibiting microRNAs," Cell Death and Differentiation, Vol. 19, 2012, pp. 937-946. doi:10.1038/cdd.2011.175

[22] A. Conigliaro, M. Colletti, C. Cicchini, M. T. Guerra, R. 
Manfredini, R. Zini, et al., "Isolation and Characterization of a Murine Resident Liver Stem Cell," Cell Death and Differentiation, Vol. 15, No. 1, 2008, pp. 123-133. doi:10.1038/sj.cdd.4402236

[23] D. Padua and J. Massague, "Roles of TGFbeta in Metastasis,” Cell Research, Vol. 19, No. 1, 2009, pp. 89-102. doi:10.1038/cr.2008.316

[24] D. Lee, Y. H. Chung, J. A. Kim, Y. S. Lee, M. K. Jang, K. M. Kim, et al., "Transforming Growth Factor Beta 1 Overexpression Is Closely Related to Invasiveness of Hepatocellular Carcinoma,” Oncology, Vol. 82, No. 1, 2012, pp. 11-18. doi:10.1159/000335605

[25] Y. Shirai, S. Kawata, S. Tamura, N. Ito, H. Tsushima, K. Takaishi, et al., "Plasma Transforming Growth FactorBeta 1 in Patients with Hepatocellular Carcinoma. Comparison with Chronic Liver Diseases," Cancer, Vol. 73, No. 9, 1994, pp. 2275-2279.

doi:10.1002/1097-0142(19940501)73:9<2275::AID-CNC R2820730907>3.0.CO;2-T

[26] N. Ito, S. Kawata, S. Tamura, Y. Shirai, S. Kiso, H. Tsushima, et al., "Positive Correlation of Plasma Transforming Growth Factor-Beta 1 Levels with Tumor Vascularity in Hepatocellular Carcinoma," Cancer Letters, Vol. 89, No. 1, 1995, pp. 45-48.

[27] N. Ito, S. Kawata, S. Tamura, K. Takaishi, Y. Shirai, S. Kiso, et al., "Elevated Levels of Transforming Growth Factor Beta Messenger RNA and Its Polypeptide in Human Hepatocellular Carcinoma," Cancer Research, Vol. 51, No. 15, 1991, pp. 4080-4083.

[28] K. Matsuzaki, M. Date, F. Furukawa, Y. Tahashi, M. Matsushita, K. Sakitani, et al., "Autocrine Stimulatory Mechanism by Transforming Growth Factor Beta in Human Hepatocellular Carcinoma," Cancer Research, Vol. 60, No. 5, 2000, pp. 1394-1402.

[29] J. Xu, S. Lamouille and R. Derynck, "TGF-Beta-Induced Epithelial to Mesenchymal Transition," Cell Research, Vol. 19, No. 2, 2009, pp. 156-172. doi:10.1038/cr.2009.5

[30] J. Fuxe, T. Vincent and A. Garcia de Herreros, "Transcriptional Crosstalk between TGF-Beta and Stem Cell Pathways in Tumor Cell Invasion: Role of EMT Promoting Smad Complexes," Cell Cycle, Vol. 9, No. 12, 2010, pp. 2363-2374. doi:10.4161/cc.9.12.12050

[31] L. Caja, E. Bertran, J. Campbell, N. Fausto and I. Fabregat, "The Transforming Growth Factor-Beta (TGF-Beta) Mediates Acquisition of a Mesenchymal Stem Cell-Like Phenotype in Human Liver Cells," Journal of Cellular Physiology, Vol. 226, No. 5, 2011, pp. 1214-1223. doi:10.1002/jcp.22439

[32] R. Derynck, R. J. Akhurst and A. Balmain, "TGF-Beta Signaling in Tumor Suppression and Cancer Progression,” Nature Genetics, Vol. 29, No. 2, 2001, pp. 117-129. doi:10.1038/ng1001-117

[33] Y. N. Kim, K. H. Koo, J. Y. Sung, U. J. Yun and H. Kim, "Anoikis Resistance: An Essential Prerequisite for Tumor Metastasis," International Journal of Cell Biology, Vol. 2012, 2012, Article ID: 306879. doi:10.1155/2012/306879

[34] J. Massague, “TGFbeta in Cancer,” Cell, Vol. 134, No. 2,
2008, pp. 215-230. doi:10.1016/j.cell.2008.07.001

[35] J. Zavadil, M. Bitzer, D. Liang, Y. C. Yang, A. Massimi, S. Kneitz, et al., "Genetic Programs of Epithelial Cell Plasticity Directed by Transforming Growth Factor-Beta," Proceedings of the National Academy of Sciences of the United States of America, Vol. 98, No. 12, 2001, pp. 6686-6691. doi:10.1073/pnas.111614398

[36] J. Zavadil and E. P. Bottinger, "TGF-Beta and Epithelialto-Mesenchymal Transitions,” Oncogene, Vol. 24, No. 37, 2005, pp. 5764-5774. doi:10.1038/sj.onc.1208927

[37] B. Ozdamar, R. Bose, M. Barrios-Rodiles, H. R. Wang, Y. Zhang and J. L. Wrana, "Regulation of the Polarity Protein Par6 by TGFbeta Receptors Controls Epithelial Cell Plasticity,” Science, Vol. 307, No. 5715, 2005, pp. 16031609. doi:10.1126/science. 1105718

[38] S. Thuault, E. J. Tan, H. Peinado, A. Cano, C. H. Heldin and A. Moustakas, "HMGA2 and Smads Co-Regulate SNAIL1 Expression during Induction of Epithelial-to-Mesenchymal Transition," The Journal of Biological Chemistry, Vol. 283, No. 48, 2008, pp. 33437-33446. doi:10.1074/jbc.M802016200

[39] B. P. Zhou, J. Deng, W. Xia, J. Xu, Y. M. Li, M. Gunduz, et al., "Dual Regulation of Snail by GSK-3beta-Mediated Phosphorylation in Control of Epithelial-Mesenchymal Transition," Nature Cell Biology, Vol. 6, No. 10, 2004, pp. 931-940. doi:10.1038/ncb1173

[40] A. Marchetti, M. Colletti, A. M. Cozzolino, C. Steindler, M. Lunadei, C. Mancone, et al., "ERK5/MAPK Is Activated by TGFbeta in Hepatocytes and Required for the GSK-3beta-Mediated Snail Protein Stabilization,” Cellular Signalling, Vol. 20, No. 11, 2008, pp. 2113-2118. doi:10.1016/j.cellsig.2008.08.002

[41] A. M. Cozzolino, T. Alonzi, L. Santangelo, C. Mancone, B. Conti, C. Steindler, et al., "TGFbeta Overrides HNF4alpha Tumor Suppressing Activity through GSK3beta Inactivation: Implication for Hepatocellular Carcinoma Gene Therapy," Journal of Hepatology, Vol. 58, No. 1, 2012, pp. 65-72. doi:10.1016/j.jhep.2012.08.023

[42] A. Miyoshi, Y. Kitajima, S. Kido, T. Shimonishi, S. Matsuyama, K. Kitahara, et al., "Snail Accelerates Cancer Invasion by Upregulating MMP Expression and Is Associated with Poor Prognosis of Hepatocellular Carcinoma,” British Journal of Cancer, Vol. 92, No. 2, 2005, pp. 252258.

[43] N. L. Lazarevich, O. A. Cheremnova, E. V. Varga, D. A. Ovchinnikov, E. I. Kudrjavtseva, O. V. Morozova, et al., "Progression of HCC in Mice Is Associated with a DownRegulation in the Expression of Hepatocyte Nuclear Factors,” Hepatology, Vol. 39, No. 4, 2004, pp. 1038-1047. doi:10.1002/hep.20155

[44] C. Yin, Y. Lin, X. Zhang, Y. X. Chen, X. Zeng, H. Y. Yue, et al., "Differentiation Therapy of Hepatocellular Carcinoma in Mice with Recombinant Adenovirus Carrying Hepatocyte Nuclear Factor-4alpha Gene,” Hepatology, Vol. 48, No. 5, 2008, pp. 1528-1539. doi:10.1002/hep.22510

[45] B. F. Ning, J. Ding, C. Yin, W. Zhong, K. Wu, X. Zeng, et al., "Hepatocyte Nuclear Factor 4Alpha Suppresses the 
Development of Hepatocellular Carcinoma,” Cancer Research, Vol. 70, No. 19, 2010, pp. 7640-7651.

doi:10.1158/0008-5472.CAN-10-0824

[46] H. Y. Yue, C. Yin, J. L. Hou, X. Zeng, Y. X. Chen, W.
Zhong, et al., "Hepatocyte Nuclear Factor 4alpha Attenuates Hepatic Fibrosis in Rats," Gut, Vol. 59, No. 2, 2010, pp. 236-246. doi:10.1136/gut.2008.174904 